\title{
Predicting the molecular subtype of breast cancer based on mammography and ultrasound findings
}

\section{S. Rashmi, S. Kamala, S. Sudha Murthy', Swapna Kotha, Y. Suhas Rao, K. Veeraiah Chaudhary}

Departments of Radiodiagnosis, 'Pathology and Lab Diagnosis, Basavatarakam Indo American Cancer Hospital and Research Institute, Hyderabad, Telangana, India

Correspondence: Dr. S. Rashmi, Consultant Radiologist, Department of Radiodiagnosis, Basvatarakam Indo American Cancer Hospital and Research Institute, Banjara Hills, Hyderabad - 500 034, India. E-mail: rashmi4210@gmail.com

\begin{abstract}
Aim: To determine the correlation between mammography and ultrasound features of breast cancer with molecular subtypes and to calculate the predictive value of these features. Materials and Method: This is a prospective study of consecutive patients with breast cancer presenting between January 2016 and July 2017, who underwent mammography and/or ultrasound of breast and excision of the breast mass. Patients with contralateral breast mass, metastases, h/o prior cancer treatment, and other malignancies were excluded. On mammography, the presence or absence of microcalcification was noted. On ultrasound examination size, margins, microcalcification, posterior acoustic features, vascularity, and axillary nodes were assessed. Margins were categorized into circumscribed and non-circumscribed. Posterior acoustic features were classified into four categories: shadowing, enhancement, mixed, and no changes. Vascularity was assessed based on Adler's index into grades $0,1,2$, and 3 . Grades 0 and 1 were considered low and 2 and 3 were high. Results: Tumors with non-circumscribed margins and posterior acoustic shadowing were likely to be luminal A or B subtype of breast cancer [odds ratio (OR) 5.78; 95\% confidence interval (Cl) 3.68-9.80; $P<0.0001$ ]. Tumors with non-circumscribed margins, posterior acoustic shadowing, and high vascularity were more likely to be luminal B subtype (OR 2.88; $95 \% \mathrm{Cl} 2-4.14 ; P-<0.0001)$. Tumors with microcalcification and posterior mixed acoustic pattern were strongly associated to be HER2-positive (OR 5.48; 95\% Cl 3.06-9.80; $P<0.0001)$. Tumors with circumscribed margins and posterior acoustic enhancement were highly suggestive of triple-negative breast cancer (OR 7.06; 95\% Cl 4.64-10.73; $P<0.0001$ ). Conclusion: Microcalcification detected on mammography and certain ultrasound features such as circumscribed or non-circumscribed margins, posterior acoustic features, and vascularity are strongly correlated in predicting the molecular subtypes of breast cancer, and thus may further expand the role of conventional breast imaging.
\end{abstract}

Key words: Breast cancer; mammography; microcalcifications; molecular subtype; posterior acoustic features; predicting value; ultrasound; vascularity

\section{Introduction}

Breast cancer is the leading cause of death due to cancer in women worldwide, and the incidence has been increasing. ${ }^{[1]}$

\begin{tabular}{|l|l|}
\hline \multicolumn{2}{|c|}{ Access this article online } \\
\hline Quick Response Code: & \\
\hline & Website: \\
\hline & www.ijri.org \\
& DOI: \\
\hline
\end{tabular}

It is a diverse group of diseases with variable natural history, histopathological subtypes, biological characteristics, and

This is an open access journal, and articles are distributed under the terms of the Creative Commons Attribution-NonCommercial-ShareAlike 4.0 License, which allows others to remix, tweak, and build upon the work non-commercially, as long as appropriate credit is given and the new creations are licensed under the identical terms.

For reprints contact: reprints@medknow.com

Cite this article as: Rashmi S, Kamala S, Murthy SS, Kotha S, Rao YS, Chaudhary KV. Predicting the molecular subtype of breast cancer based on mammography and ultrasound findings. Indian J Radiol Imaging 2018;28:354-61. 
response to treatments. The molecular subtyping of breast cancer has become an essential requirement for treatment planning, disease prognosis, and to avoid overtreatment. ${ }^{[2]}$ The St. Gallen International Expert Consensus recently classified breast cancer into five different molecular subtypes based on gene expression patterns: luminal A (LA), luminal B [(LB; HER2-), LB (HER2+)], human epidermal growth factor receptor 2 (HER2)-enriched, and basal-like (triple-negative). Pathologically, these molecular subtypes are categorized based on tumor markers' expression status: estrogen receptor (ER), progesterone receptor (PR), HER2neu overexpression, and Ki-67 index. Invasive breast cancer with ER- and/or PR-positive and low Ki-67 index (ki-67 <14\%) are considered LA type, ER- and/or PR-positive with high Ki-67 index (Ki-67 $\geq 14 \%$ ) and HER2-negative are LB (HER2-) subtype, ER- and/or PR-positive with HER2-positive are LB (HER2+) subtype, ER- and PR-negative with HER2neu overexpression are HER2-enriched type, and breast cancer with all three receptors (ER/PR/HER2neu) negative are basal or triple-negative type ${ }^{[3-8]}$ Immunohistochemistry (IHC) is the gold standard for detecting hormone receptor (ER/PR), HER2 overexpression, and Ki-67 expression status, but it is an invasive method, expensive test, and not readily available in many of the developing and underdeveloped countries. Mammography and ultrasound are the primary imaging modalities used for breast cancer screening, diagnosis of breast cancer, staging, treatment response assessment, and follow-up of the treated breast cancer patients. A few retrospective studies done in the past found that ultrasound features have high predictive values in differentiating triple-negative breast cancer (TNBC) and non-triple-negative breast cancer and also in determining the grades of tumor. ${ }^{[5-11]}$ A study done by Cen et al. has shown good correlation between the mammographically detected suspicious microcalcifications and HER2 overexpression status. ${ }^{[12]}$ To the best of our knowledge, there is no published study which has shown correlation between each of the five molecular subtypes of breast cancer and conventional breast imaging features. Therefore, the aim of this study was to correlate the mammography and ultrasound features with molecular status of breast cancer and also to calculate the predictive value of their correlation.

\section{Materials and Methods}

This is a prospective study conducted at a tertiary care cancer institute, and institutional ethics committee board approved the study protocol. The study sample consisted of 191 consecutive symptomatic patients presenting between January 2016 and July 2017 who were evaluated with mammography and/or ultrasonography (USG) and underwent surgical excision followed by histopathological and IHC examination of the sample. Patients with history of prior neoadjuvant chemotherapy, prior cancer treatment, bilateral breast cancers, and those unable to undergo histopathological and IHC examinations were excluded.
Image analysis

Among patients who underwent digital mammography, presence or absence of microcalcifications was noted. All the mammography examinations were supplemented with ultrasound of the breasts. All the ultrasound scans were performed by one of the four trained breast radiologists on Siemens Acuson S2000 ultrasound system with linear transducer of 18-MHz frequency. Patients less than 40 years of age were primarily evaluated with ultrasound of breast and some of them were complemented with mammography in case of doubt or after diagnosis. On USG, tumor size, shape, margins, echogenicity, posterior acoustic features, and vascularity on power Doppler were assessed. All the ultrasound images were independently analyzed by each radiologist, and in cases of interobserver disagreement, a joint consensus was reached after second observation. All the breast radiologists were blinded to the receptor status of breast masses. The imaging features' analysis in this study is illustrated and defined in Table 1.

\section{Histopathological and IHC analysis}

The formalin-fixed and paraffin-embedded tissue samples were examined by experienced pathologists for tumor type and grading, and subsequently for immunohistochemical stain with appropriate antibodies to assess ER/PR status, Ki-67 index, and HER2 expression. On IHC examination, the ER or PR status was considered positive when the nuclear staining was $\geq 1 \%$. Ki- 67 index $<14 \%$ was considered as low expression and $\geq 14 \%$ was considered high expression. HER2 expressions with grades 0 and $1+$ were considered negative, grade $2+$ was equivocal, and grade $3+$ was considered positive [Figure 1A-D]. All the equivocal samples were further analyzed with fluorescence in situ hybridization technique and were considered positive if the samples showed $>2$-fold increase for gene amplification. Based on ER/PR/HER2 and Ki-67 expression status, breast cancers were categorized into five molecular subtypes: LA subtype: ER- and/or PR-positive, HER2-negative, and Ki-67 <14\%; LB (HER2-) subtype: ER- and/or PR-positive,

Table 1: Imaging features characteristics

\begin{tabular}{ll}
\hline $\begin{array}{l}\text { Imaging features } \\
\text { Microcalcifications }\end{array}$ & Present or absent \\
Size & $\begin{array}{l}\text { Maximum dimension on ultrasound } \\
\text { Circumscribed or non-circumscribed } \\
\text { (spiculated/angular/microlobulated) }\end{array}$ \\
Oval/round/irregular \\
Shape & Parallel/non-parallel \\
Orientation & Anechoic/hypoechoic/isoechoic/hyperechoic \\
Echogenicity & Shadowing/enhancement/mixed/no changes \\
Posterior acoustic feature & Echogenic halo or abrupt interface \\
Lesion boundary & Displacement or disruption \\
Cooper's ligament & Adler's degree $0, \mathrm{I}=$ low; II and III=high \\
Vascularity & $0=$ no vascularity \\
& $\mathrm{I}=$ minimal \\
& II=moderate \\
III=marked
\end{tabular}


HER2-negative, and Ki-67 $\geq 14 \%$; LB (HER2+) subtype: ER- and/or PR-positive and HER2-positive; HER2-enriched type (HER2): ER- and PR-negative and HER2-positive; triple-negative type (TN): ER, PR, and HER2-negative.

\section{Statistical analysis}

Our data was collected on Microsoft Office Excel 2010 and statistical analysis was performed using IBM SPSS version 20. The imaging features of different molecular subtypes were compared using univariate and multivariate analyses of data. For all the tests, statistical significance was assumed when $P$ value $<0.05$. Receiver operating characteristic (ROC) curves were also drawn and area under the curve was calculated.

\section{Results}

Demographic and imaging features of different molecular subtypes of breast cancers are summarized in Table 2.

Our study cohort consisted of 191 patients with a mean age of $52 \pm 11$ years (range, 24-85 years); 41 (21\%) were LA; 45 (23\%) were LB; 24 (13\%) were LB (HER2+); 30 (16\%) were HER2-enriched; and 51 (27\%) were TNBC types [Figures 2 and 3].

Age distribution and imaging appearances of each molecular subtype are summarized in Figure 4A-F. The mean age for TNBC was significantly less than the other molecular types (46 vs 52 years). The mean tumor size measured on ultrasound was $32 \pm 11 \mathrm{~mm}$ and the majority of them in each molecular subtype was $>20 \mathrm{~mm}$. The mean size was relatively higher because only symptomatic patients were included in the study. Breast cancers with microcalcifications were more commonly seen in HER2-positive lesions than HER2-negative (67\% vs 33\%). Most breast cancers had non-circumscribed margins (80\%)

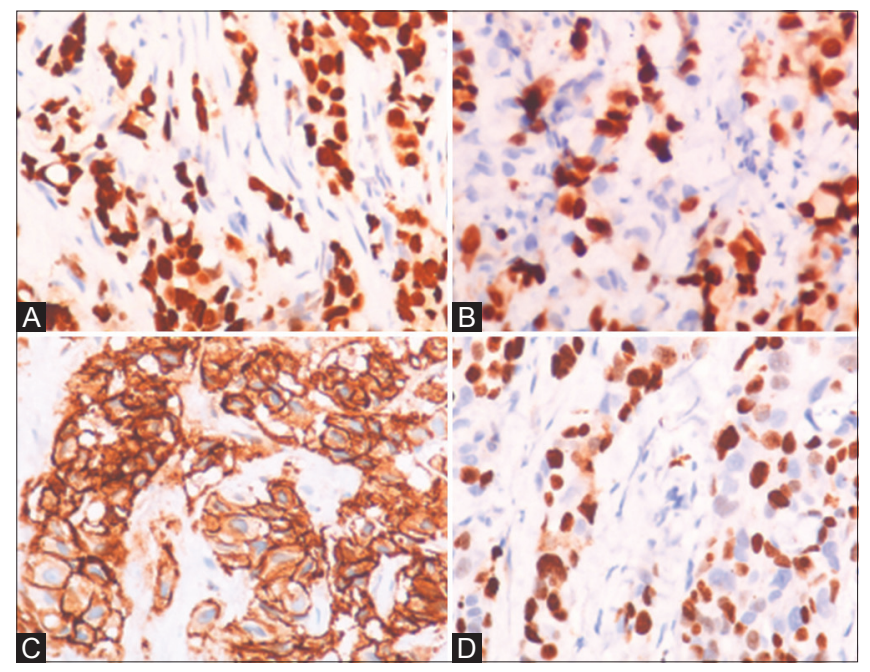

Figure 1 (A-D): Immunohistochemistry pictures of (A) Positive estrogen receptor. (B) Positive progesterone receptor. (C) HER2 neu (score3+). (D) ki67 index and lesions with circumscribed margins were more likely to be triple-negative type (63\% vs 37\%). Posterior shadowing was predominantly seen with LA and LB subtypes than the others ( $86 \%$ vs $14 \%$ ), and LB tumors showed high vascularity compared with LA (91\% vs $12 \%)$.

Multivariate analysis with predictive value of imaging features with each molecular subtype is shown in Table 3.

Breast tumors with circumscribed tumor margins on ultrasound were strongly associated with TNBC type [odds ratio $(\mathrm{OR}) 8 ; P<0.0001]$ when compared with other subtypes. Posterior acoustic features' analysis suggested that tumors with posterior shadowing were more likely to be LA or LB type (OR 6.2 and 4.2; $P<0.0001$ ); tumors with mixed posterior acoustic feature were significantly associated with HER2+ overexpression (OR 3.97; $P=0.0012$ ); and tumors with posterior enhancement were strongly associated with TNBC (OR 12.7; $P<0.0001$ ). Mammographically detected microcalcifications were strongly associated with HER2 overexpression (OR 8.1; $P<0.0001)$. On analyzing the vascularity on power Doppler, tumors with high Adler degree of vascularity (II or III) were better associated with LB type, that is, ER- and/or PR-positive with high ki-67 index (OR 12.48; $P<0.0001$ ) or HER2-enriched type (OR 2.24; $P=0.058$ ). LA and triple-negative type of tumors were found more likely to have low vascularity (Adler degree 0 or I).

On combining more than one imaging feature, breast tumors with non-circumscribed margins and posterior shadowing were strongly associated with LA or B types (OR 5.77; $P<0.0001$ ), and if these tumors showed Adler high vascularity (grade II or III), then it was more likely to be LB (OR 2.88; $P<0.0001$ ) type of invasive breast carcinoma. Tumors with posterior mixed acoustic pattern and microcalcifications were highly associated to have HER2 overexpression (OR 5.48; $P<0.0001$ ), and tumors with circumscribed margins with posterior enhancement were very strongly associated with TNBC (OR 7.06; $P<0.0001)$.

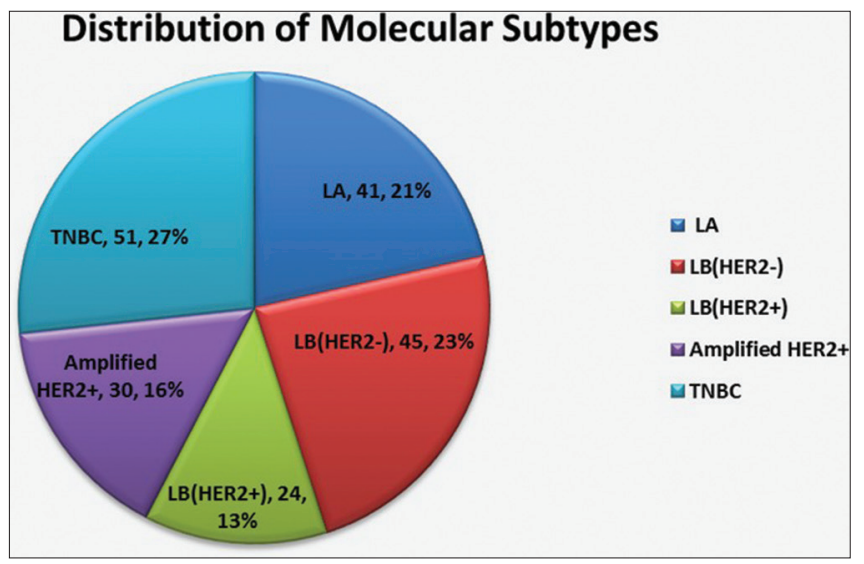

Figure 2: Pie chart showing distribution of five molecular subtypes 
ROC curves with area under the curve of each molecular subtype are illustrated in Figure 5A-C.

\section{Discussion}

In the current practice of breast cancer management, treatment is adjusted according to the receptor status determined on IHC of the particular tumor, which is the gold standard test and requires tissue sample for testing. Hence, various studies are being performed to determine how various imaging modalities including mammography, ultrasound, and magnetic resonance imaging could predict the molecular subtype of a breast tumor, which would further improve the potential for presurgical
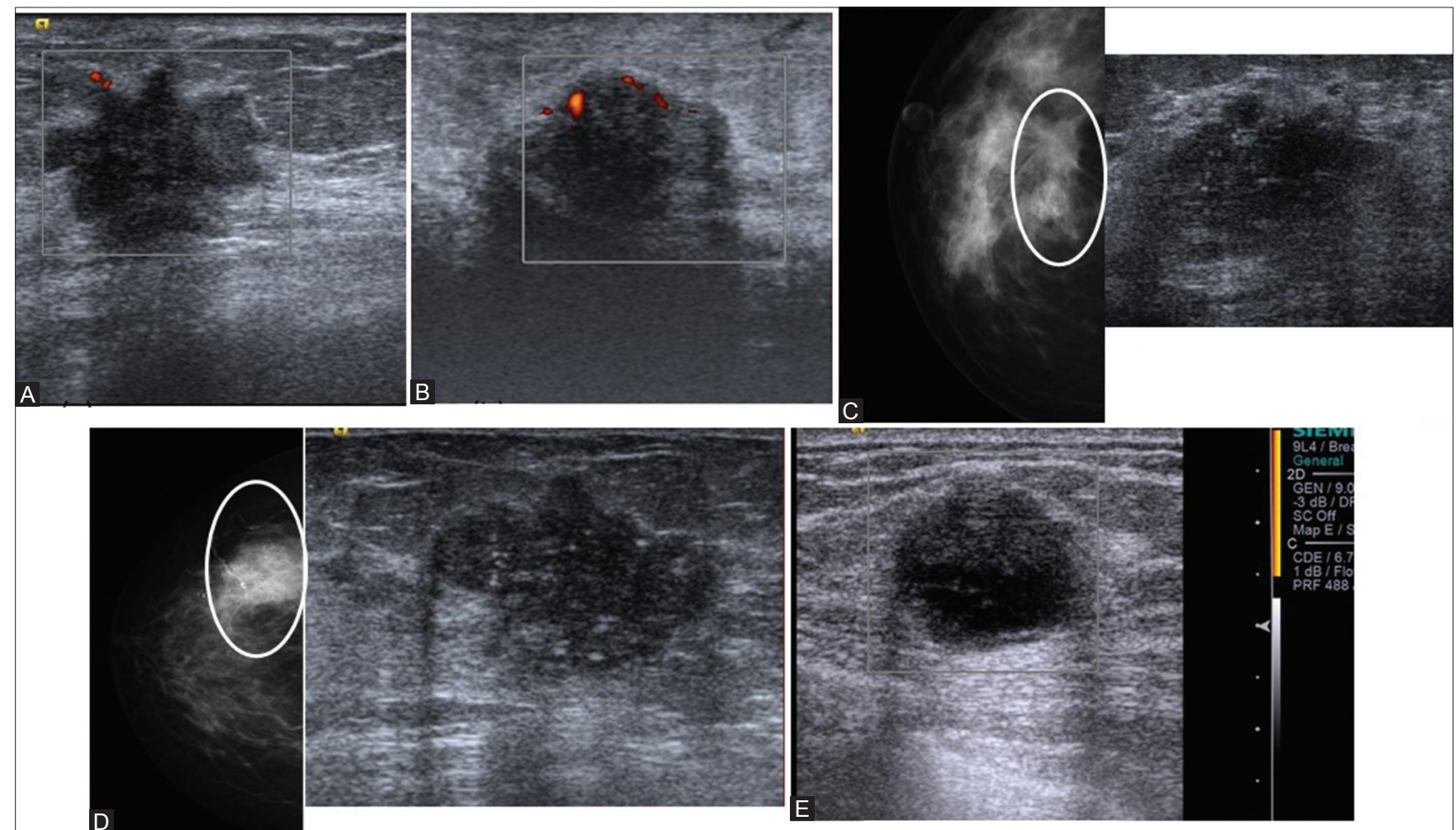

Figure 3 (A-E): Mammography, ultrasound and doppler features of five molecular subtypes: (A) LA type: Non-circumscribed mass with spiculated margins, posterior shadowing and minimal vascularity. (B) LB (her2-) type: Non-circumscribed mass with posterior shadowing and high vascularity. (C) LB (her2+) type: Non-circumscribed mass with microcalcifications and posterior shadowing. (D) HER2 enriched-type: Non-circumscribed mass with microlobulated margins, microcalcifications and posterior mixed acoustic pattern. (E) Triple negative(TN)-type: Well circumscribed mass with posterior enhancement and absent vascularity (grade 0)

Table 2: Distribution of demographic and imaging parameters of each molecular subtype of breast cancer

\begin{tabular}{|c|c|c|c|c|c|c|}
\hline Dependent variables & Total case $(n=191)$ & $\mathrm{LA}(n=41)$ & LB (her2-) $n=45$ & LB (her2+) $n=24$ & HER2enriched $(n=30)$ & $\operatorname{TNBC}(n=51)$ \\
\hline Mean age & $51.32 \pm 11.33$ & $53.29 \pm 11.60$ & $53.06 \pm 11.82$ & $53.08 \pm 11.28$ & $53.43 \pm 10.01$ & $45.91 \pm 10.08$ \\
\hline Micro-calcifications on mammogram & $57(29.84 \%)$ & $12(29.26 \%)$ & $3(6.67 \%)$ & $17(70.83 \%)$ & $21(70 \%)$ & $4(7.84 \%)$ \\
\hline \multicolumn{7}{|l|}{ Tumour size on USG } \\
\hline$<20 \mathrm{~mm}$ & $37(19.37 \%)$ & $5(12.20 \%)$ & $13(28.89 \%)$ & $3(12.5 \%)$ & $6(20 \%)$ & $10(19.61 \%)$ \\
\hline$>20 \mathrm{~mm}$ & $154(80.63 \%)$ & $36(87.80 \%)$ & $32(71.11 \%)$ & $21(87.5 \%)$ & $24(80 \%)$ & $41(80.39 \%)$ \\
\hline \multicolumn{7}{|l|}{ Margins } \\
\hline Non-circumscribed & $153(80.1 \%)$ & $39(95.12 \%)$ & $41(91.11 \%)$ & $21(87.5 \%)$ & $25(83.33 \%)$ & $27(52.94 \%)$ \\
\hline Circumscribed & $38(19.9 \%)$ & $2(4.88 \%)$ & $4(8.89 \%)$ & $3(12.5 \%)$ & $5(16.67 \%)$ & $24(47.06 \%)$ \\
\hline \multicolumn{7}{|l|}{ Posterior acoustic features } \\
\hline Shadowing & $81(42.40 \%)$ & $31(75.61 \%)$ & $31(68.89 \%)$ & $9(37.5 \%)$ & $5(16.67 \%)$ & $5(9.80 \%)$ \\
\hline Enhancement & $61(31.93 \%)$ & $1(2.43 \%)$ & $5(11.11 \%)$ & $1(4.16 \%)$ & $12(40 \%)$ & $42(83.4 \%)$ \\
\hline Mixed & $39(20.42 \%)$ & $2(4.87 \%)$ & $8(17.78 \%)$ & $13(54.17 \%)$ & $13(43.33 \%)$ & $3(5.9 \%)$ \\
\hline No changes & $10(5.23 \%)$ & $8(19.51 \%)$ & $1(2.22 \%)$ & $1(4.16 \%)$ & 0 & 0 \\
\hline \multicolumn{7}{|l|}{ Adler's vascularity index } \\
\hline High (grade II \& III) & $88(46.07 \%)$ & $36(87.80 \%)$ & $4(8.9 \%)$ & $2(8.3 \%)$ & $9(30 \%)$ & $37(72.5 \%)$ \\
\hline High (grade II \& III) & $103(53.93$ & $5(12.20 \%)$ & $41(91.1 \%)$ & $22(91.7 \%)$ & $21(70 \%)$ & $14(27.5 \%)$ \\
\hline
\end{tabular}



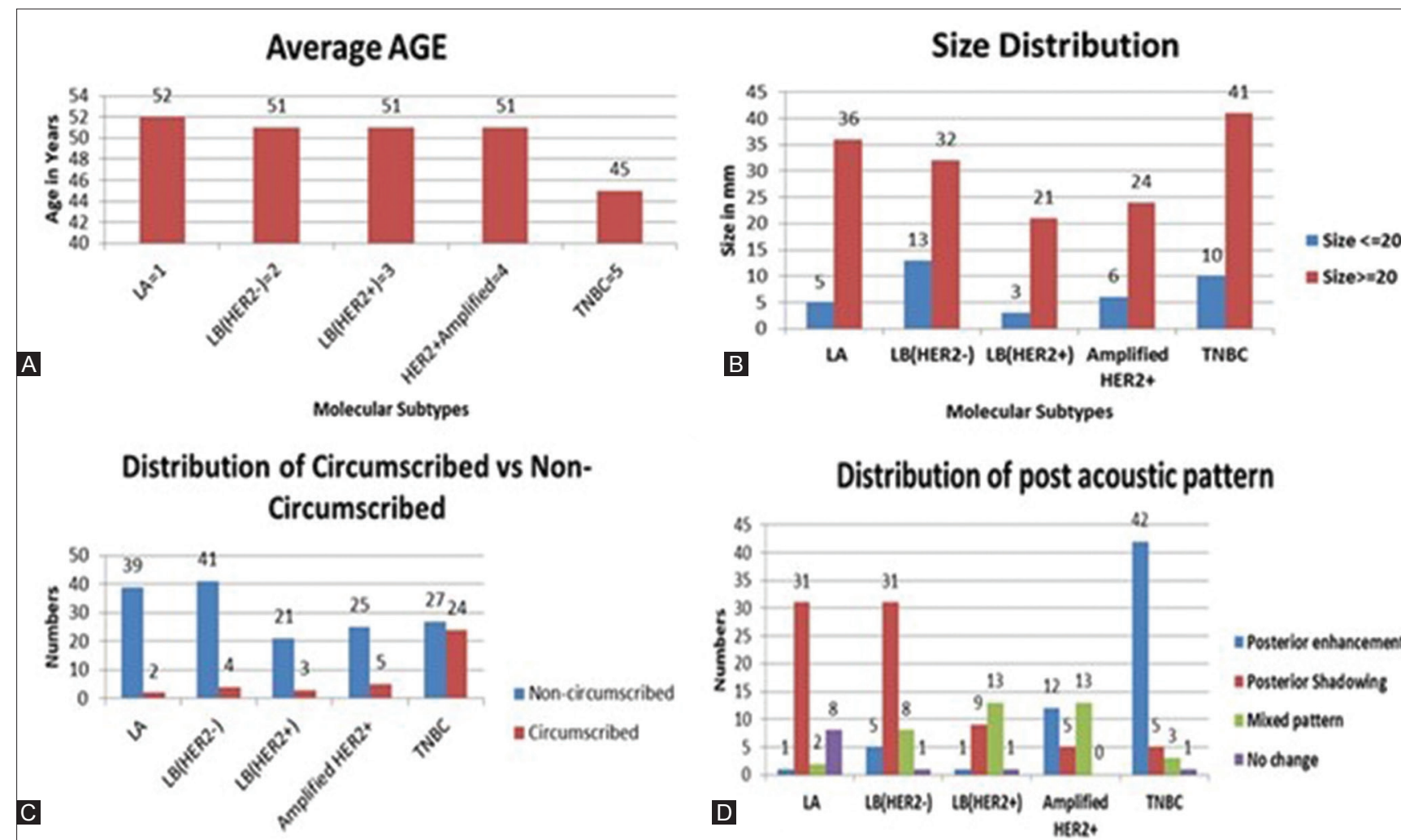

Non-circumscribed n Circumscribed

C

Molecular Subtypes

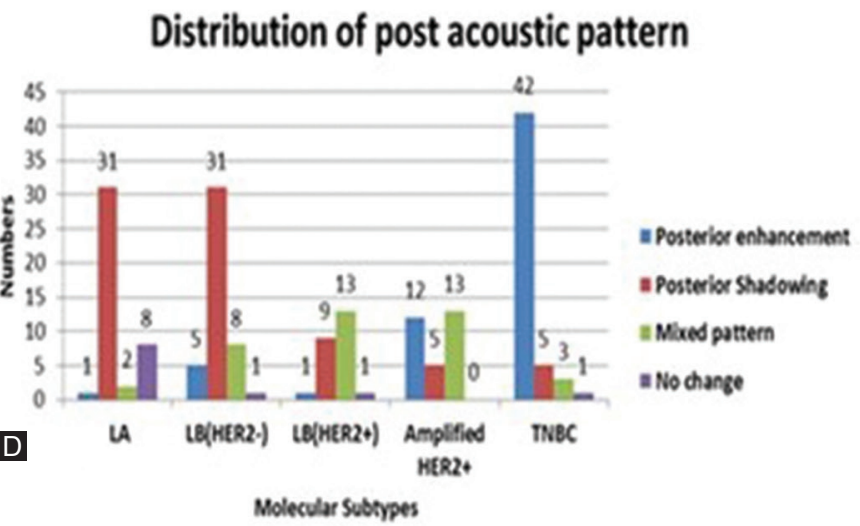

\section{Distrubtion of Microcalcification}

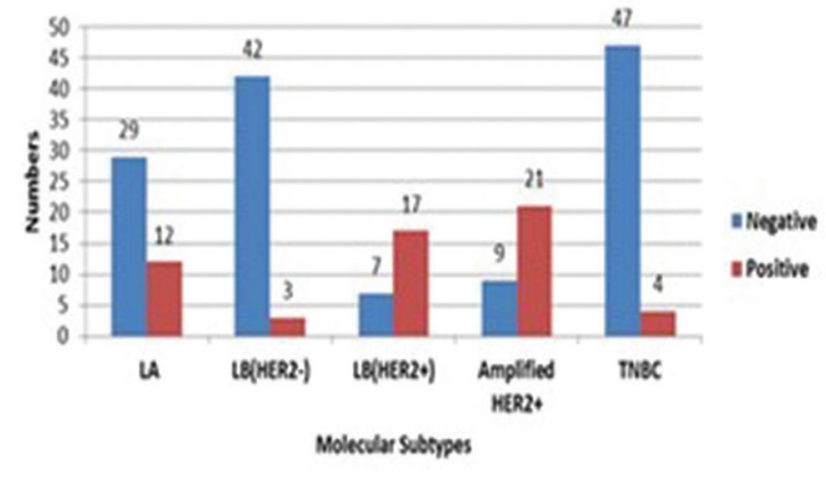

E

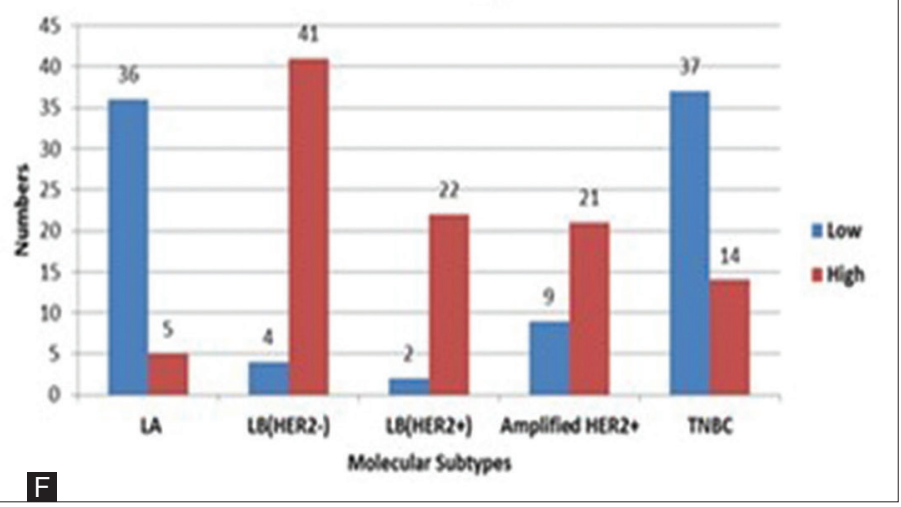

Figure 4 (A-F): Age distribution and imaging characteristics of each molecular subtype: (A) Mean age of TNBC was significantly less than the other subtypes. (B) Majority of tumors in each subtype were more than $20 \mathrm{~mm}$. (C) Circumscribed tumors were more likely to be TNBC type. (D) Posterior shadowing was commonly seen in LA and LB subtype, and posterior enhancement was seen in TNBC type. (E) Microcalcifications were most commonly present in HER2+ tumors. (F) LB and HER2 type were more vascular compared to others

treatment strategies. Mammography and ultrasound are the primary imaging modalities used for evaluation of breast cancer. In this study, we analyzed the associations between mammography and ultrasound features with molecular subtypes of breast cancer categorized based on receptor status (ER, PR, HER2+, and Ki67) determined by IHC. LA and LB tumors (ER- and/or PR-positive and HER2-negative) showed significant positive association of having non-circumscribed margins and posterior acoustic shadowing on ultrasound. These findings were similar to a study done by Anupama et al. who reported that tumors with posterior shadowing had 25 times higher chances and tumors with non-circumscribed margins had 9.5 times higher chances of having hormone receptor positivity ${ }^{[14]}$ Irshad et al. also found tumors with posterior acoustic shadowing to have greater than nine times higher association with hormone receptor positivity. ${ }^{[6]}$ Similarly, Celebi et al. found that tumors with combined findings of non-circumscribed margins and posterior shadowing were found to have 10.58 times higher association with LA and LB subtypes. ${ }^{[2]}$ The presence of receptor status has a favorable prognosis as they show hormone sensitivity 

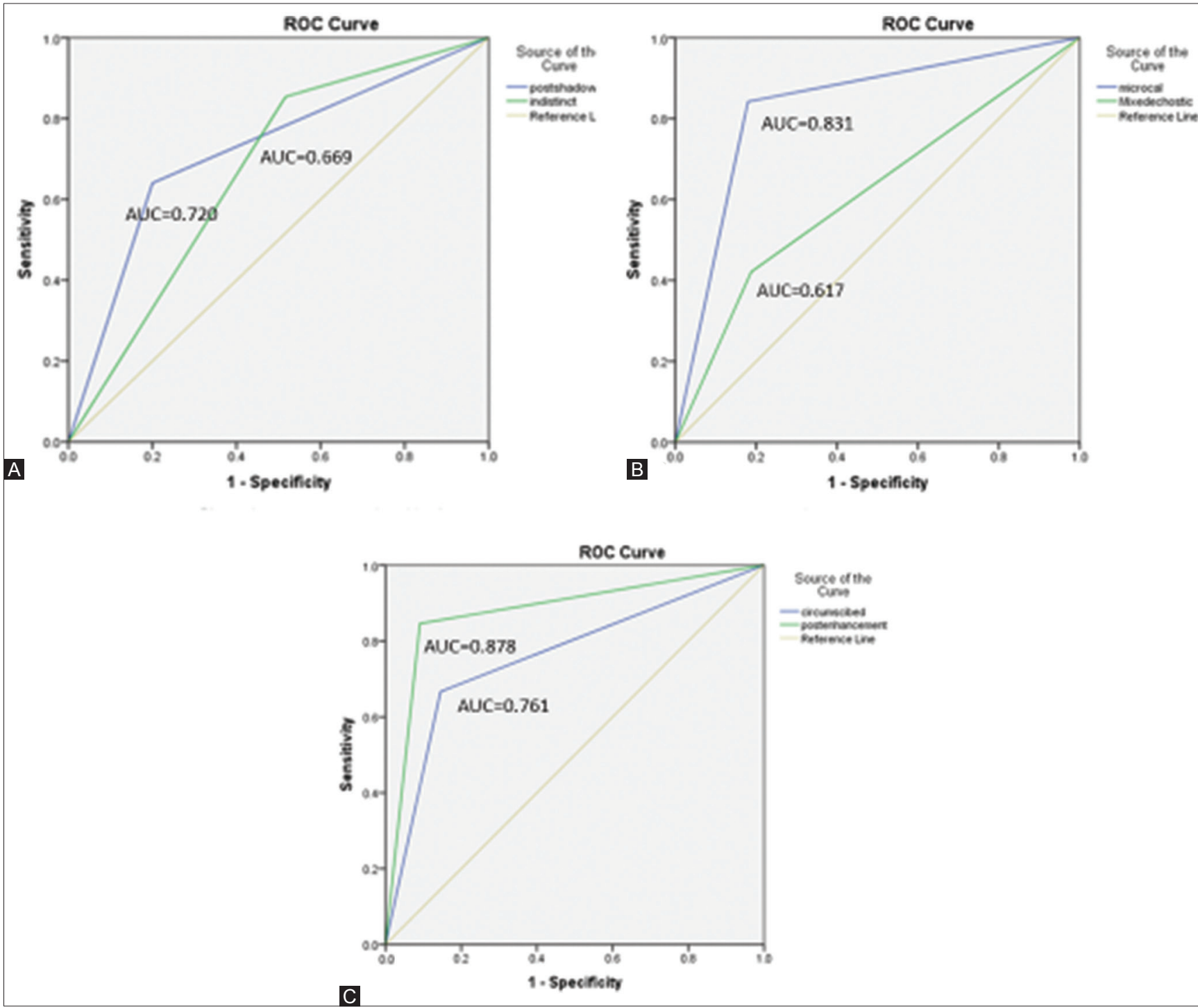

Figure $5(A-C)$ : ROC curves with significant area under the curves $(A)$ Luminal $A$ and Luminal $B$ type with posterior shadowing $(A U C=0.720)$ and non-circumscribed margins (AUC=0.669). (B) HER2 enriched tumours with microcalcification detected on mammogram $(A U C=0.831)$ and posterior mixed acoustic feature on ultrasound $(A U C=0.617)$. $(C)$ Triple negative type with posterior enhancement $(A U C=0.878)$ and non-circumscribed margins $(A \cup C=0.761)$

Table 3: Multivariate analysis with predictive value of imaging features

\begin{tabular}{|c|c|c|c|c|}
\hline Imaging features & Outcome characteristic & Odds tio (OR) & $95 \% \mathrm{Cl}$ & $P$ \\
\hline Microcalcification & HER2+vs. Others & 8.10 & 3.41 to 19.23 & $<0.0001$ \\
\hline \multirow[t]{2}{*}{ Posterior shadowing } & LA vs. Others & 6.2 & 2.81 to 13.65 & $<0.0001$ \\
\hline & LB (HER2-) vs. others & 4.25 & 2.07 to 8.71 & \\
\hline Posterior enhancement & TNBC vs. Others & 12.72 & 8.48 to 19.42 & $<0.0001$ \\
\hline Mixed pattern & HER2 vs. Others & 3.97 & 1.72 to 9.15 & 0.0012 \\
\hline Circumscribed vs. Non-circumscribed & TNBC vs. Others & 8 & 3.67 to 17.43 & $<0.0001$ \\
\hline Posterior shadowing + Noncircumscribed & LA or LB vs. others & 5.78 & 3.68 to 9.06 & $<0.0001$ \\
\hline Noncircumscribed + shadowing + high vascularity & LB vs. Others & 2.88 & 2 to 4.14 & $<0.0001$ \\
\hline Microcalcification + posterior mixed acoustic pattern & HER2+ & 5.48 & 3.06 to 9.80 & $<0.0001$ \\
\hline Circumscribed + posterior enhancement & TNBC vs. Others & 7.06 & 4.64 to 10.73 & $<0.0001$ \\
\hline
\end{tabular}

and show stromal reaction, perilesional spiculations, and fibrosis resulting in non-circumscribed margins and posterior shadowing features on ultrasound. ${ }^{[2,14]}$ In contrast to LA, LB subtype was found to have Adler higher 
degree (II and III) of vascularity. A study done by Zhang et al. correlated sonographic features with breast cancer molecular subtypes by ensemble decision and found similar results that the majority of LB subtype of tumors were found to have Adler high degree (II or III) of vascularity. ${ }^{[13]}$ Tumors detected to have microcalcifications on mammogram were strongly associated with HER2 overexpression. Seo et al. and Zhang et al. found that calcifications detected on ultrasound were more frequent in HER2-enriched tumors than the other subtypes, although some of the tumors with microcalcifications might have been missed as ultrasound is not as sensitive as mammogram for detecting microcalcification ${ }^{[13,16]}$ Cen et al. and Patel et al. also found that HER2-enriched tumors were more likely to have heterogeneous and pleomorphic microcalcifications on mammogram. ${ }^{[12,17]}$ Cen et al. also demonstrated that amorphous and heterogeneous coarse calcifications were associated with a higher incidence of LA subtype. In this study, LA was the next most common molecular subtype after HER-enriched to show microcalcification. On analyzing the posterior acoustic pattern, Her2-enriched tumors frequently demonstrated either posterior enhancement or mixed (enhancement and shadowing) feature possibly due to presence of microcalcification. This tumor type also showed higher degree of vascularity than LA, LB (HER2-negative), and TNBC subtypes. These findings were similar to previous studies published in literature. ${ }^{[2,15]}$

Tumors with well-circumscribed margins and posterior enhancement were strongly suggestive of TNBC type of breast cancer, which is the most aggressive type of breast cancer with rapid growth and necrosis. ${ }^{[12-15]} \mathrm{A}$ study done by Anupama et al. found that triple-negative cancers were hypervascular compared with non-triple-negative cancers. But our study revealed that this molecular type was less vascular than LB and HER2-enriched tumor, possibly due to more necrosis. ${ }^{[15,18]}$ TNBCs were more frequent in younger age group and were negatively associated with microcalcifications. ${ }^{[2,6,14]}$

Receptor status is detected on IHC test, an invasive test, which can cause procedure-related discomfort and complications in a patient. The reliability of the test also depends on the handling and processing of the tissue, which can occasionally lead to false-negative results. These tests are very expensive and not widely available in many developing and underdeveloped countries. On the other hand, mammography and ultrasound are noninvasive technologies, readily available, and used as the primary imaging modalities for breast cancer evaluation. Knowing the predictive value of certain imaging modalities may help the radiologists and clinicians in stratifying their patients enabling them to manage according to the resources available to them. If imaging features are highly suggestive of positive receptor status, it can further help the clinician in deciding the therapy in cases of discordance.

The limitations of our study were small sample size and non-inclusion of all imaging features of BI-RADS lexicon.

\section{Conclusion}

Microcalcification detected on mammography and certain ultrasound features such as tumor margins, posterior acoustic features, and vascularity on Doppler are strongly correlated in predicting the molecular subtype of breast cancer, and thus may further expand the role of conventional breast imaging for more precise diagnosis of breast cancer. Tumors with non-circumscribed margins and posterior shadowing are predicted to be LA or LB subtype. LB subtype cancers are more vascular than the LA subtype. Tumors with microcalcification, posterior mixed acoustic feature, and high vascularity are strongly predicted to be HER2 subtype. Circumscribed tumors with posterior enhancement and absence of microcalcification are predicted to be triple-negative type of breast cancer. However, larger multicenter studies are recommended for validating this.

Financial support and sponsorship

Nil.

Conflicts of interest

There are no conflicts of interest.

\section{References}

1. Ferlay J, Soerjomataram I, Dikshit R, Eser S, Mathers C, Rebelo M, et al. Cancer incidence and mortality worldwide: Sources, methods and major patterns in GLOBOCAN 2012. Int J Cancer 2015:136:359-86.

2. Celebi F, Pilanci KN, Ordu C, Agacayak F, Alco G, Ilgun S, et al. The role of ultrasonographic findings to predict molecular subtype, histologic grade, and hormone receptor status of breast cancer. Diagn Interv Radiol 2015;21:448-53.

3. Yaganawa M, Ikemot K, Kawauchi S. Luminal A and Luminal B (HER2 negative) subtypes of breast cancer consist of a mixture of tumors with different genotype. BMC Res Notes 2012;5:376.

4. Huber KE, Carey LA, Wazer DE. Breast cancer molecular subtypes in patients with locally advanced disease: Impact on prognosis, patterns of recurrence, and response to therapy. Semin Radiat Oncol 2009;19:204-10.

5. Lam SW, Jimenez CR, Boven E. Breast cancer classification by proteomic technologies: Current state of knowledge. Cancer Treat Rev 2014;40:129-38.

6. Irshad A, Leddy R, Pisano E, Nathaniel Baker, Madelene Lewis, Susan A, et al. Assessing the role of ultrasound in predicting the biological behavior of breast cancer. AJR Am J Roentgenol 2013;200:284-90.

7. Blaichman J, Marcus JC, Alsaadi T, El-Khoury M, Meterissian S, Mesurolle B, et al. Sonographic appearance of ductal carcinoma of the breast according to histologic grade. AJR Am J Roentgenol 2012;199:W402-8.

8. Reis-Filho JS, Simpson PT, Gale T, Lakhani SR. The molecular 
genetics of breast cancer: The contribution of comparative genomic hybridization. Pathol Res Pract 2005;201:713-25.

9. Bosch A, Eroles P, Zaragoza R, Vina JR, Lluch A. Triple negative breast cancer: Molecular features, pathogenesis, treatment and current lines of research. Cancer Treat Rev 2010;36:206-15.

10. Wang Y, Ikeda DM, Narasimhan B, Longacre TA, Bleicher RJ, Pal S, et al. Estrogen receptor-negative invasive breast cancer: Imaging features of tumors with and without human epidermal growth factor receptor type 2 overexpression. Radiology 2008;246:367-75.

11. Cheang MB, Chia SK, Voduc D, Gao D, Leung S, Snider J, et al. Ki67 index, HER2 status, and prognosis of patients with Luminal B breast cancer. J Natl Cancer Inst 2009;101:736-50.

12. Cen D, Xu L, Li N, Chen Z, Wang L, Zhou S, et al. BI-RADS 3-5 microcalcifications can preoperatively predict breast cancer HER2 and Luminal a molecular subtype. Oncotarget 2017;8:13855-62.

13. Zhang L, Li J, Xiao Y, Cui H, Du G, Wang Y, et al. Identifying ultrasound and clinical features of breast cancer molecular subtypes by ensemble decision. Sci Rep 2015;5:11085.
14. Anupama T, Prachi S, Smita M, Neelam W, Natasha G, Navneet K, et al. Role of Sonography in predicting the hormone receptor status of breast cancer: A prospective study. J Diagn Med Sonogr 2017;1-12.

15. Boisserie-Lacroix M, Mac Grogan G, Debled M, Ferron S, Asad-Syed M, Brouste V, et al. Radiological features of triple negative breast cancers (about 73 cases). Diagn Interv Imaging 2012;93:196-203.

16. Seo BK, Pisano ED, Kuzimak CM, Koomen M, Pavic D, Lee $\mathrm{Y}$, et al. Correlation of HER-2/ne overexpression with mammography and age distribution in primary breast carcinomas. Acad Radiol 2006;13:1211-8.

17. Patel TA, Puppala M, Ogunti RO, Ensor JE, He T, Shewale JB, et al. Correlating mammographic and pathologic findings in clinical decision support using natural language processing and data mining methods. Cancer 2017;123:114-21.

18. Kojima Y, Tsunoda H. Mammography and ultrasound features of triple-negative breast cancer. Breast Cancer 2011;18:146-51. 